Research Article

\title{
Typhoon Maria Precipitation Retrieval and Evolution Based on the Infrared Brightness Temperature of the Feng-Yun 4A/Advanced Geosynchronous Radiation Imager
}

\author{
Gen Wang $\mathbb{D}^{1,},{ }^{1,2}$ Kefu Wang, ${ }^{3}$ Wei Han $\mathbb{D}^{4},{ }^{4}$ Dongyong Wang $\mathbb{D},{ }^{1}$ and Xuexing Qiu $\mathbb{D}^{1}$ \\ ${ }^{1}$ Anhui Meteorological Observatory, Key Lab of Strong Weather Analysis and Forecast, Hefei, Anhui 230031, China \\ ${ }^{2}$ Anhui Institute of Meteorological, Anhui Key Lab of Atmospheric Science and Satellite Remote Sensing, Hefei, \\ Anhui 230031, China \\ ${ }^{3}$ Anhui Meteorological Bureau, Hefei, Anhui 230031, China \\ ${ }^{4}$ National Meteorological Center of China, Numerical Weather Prediction Center of China Meteorological Administration, \\ Beijing 100081, China \\ Correspondence should be addressed to Wei Han; hanwei@cma.gov.cn
}

Received 12 August 2019; Accepted 29 November 2019; Published 19 February 2020

Academic Editor: Yoshihiro Tomikawa

Copyright (c) 2020 Gen Wang et al. This is an open access article distributed under the Creative Commons Attribution License, which permits unrestricted use, distribution, and reproduction in any medium, provided the original work is properly cited.

Recognizing the importance and challenges inherent in the remote sensing of precipitation in typhoon monitoring, a study of the Advanced Geosynchronous Radiation Imager (AGRI) data from Feng-Yun 4A on typhoon precipitation was conducted. First, Typhoon Maria was selected to statistically analyze the AGRI infrared brightness temperature in the "precipitation" and "nonprecipitation" channels of the field of view. When there was precipitation, the brightness temperature of the AGRI channel changed significantly. Second, the shrunken locally linear embedding algorithm (SLLE) was adopted to carry out the retrieval of precipitation based on the brightness temperatures of AGRI infrared channels 8-14. The contribution rate of the brightness temperature at different channels to the objective function of precipitation retrieval model was obtained by the Bayesian model averaging (BMA). Based on the preliminary experimental "quantification" evaluation index, we concluded that the method adopted in this paper can be used to retrieve precipitation in infrared data and to retrieve the spiral cloud rain bands of a typhoon. Finally, based on the AGRI channel brightness temperature of a 10.8-micron window channel, we applied the membership degree information of a typhoon's dominant cloud system from the fuzzy $c$-means (FCM) clustering method to modify precipitation retrieval results. The results were used to obtain the main morphological structure of typhoon precipitation. By further analyzing the temporal variation of dominant cloud system development using the FCM method, we concluded that the brightness temperature gradient can assist in the analysis of the variation of a typhoon's intensity. This method can be applied to the continuous retrieval of large-scale precipitation. Precipitation retrieval via the AGRI can yield indicators for typhoon precipitation warnings and forecasts, thus providing a reliable reference tool for disaster prevention and mitigation.

\section{Introduction}

Precipitation plays an important role in the global energy and water cycle, and monitoring and forecasting it is crucial when a typhoon, rainstorm, or severe convection develops and occurs. China is the country that is most affected by typhoons; it is hit by an average of about seven typhoons every year. Typhoons are mainly caused by gales, rainstorms, and storm surges, but compared with typhoons caused by gales, those caused by rainstorms cause more disasters [1]. For example, after the severe Tropical Storm Bilis made landfall in the Fujian province on July 12, 2006, it led to heavy rains in the Hunan, Jiangxi, and Guangdong provinces, which caused serious landslides and mud-rock flows. The disaster caused more than 600 deaths and direct economic losses of about 4.4 billion U.S. dollars [1]. Therefore, quantitative research on typhoon precipitation is an important component of disaster prevention and reduction. 
Due to the insufficient density of ground rain gauges and the ground-based rain measuring radar observation network, it is difficult to monitor and track rainstorms and other catastrophic weather systems, not to mention the spatial distribution and changes in the intensity of typhoon precipitation [2]. Meteorological satellites can effectively obtain information such as brightness temperature in unattended areas, such as oceans, mountains, and deserts. The precipitation products obtained through retrieval have become the only effective means to cover global precipitation observation [3]. Satellite remote sensing can be divided into active microwave remote sensing, passive microwave remote sensing, and visible/infrared remote sensing. Active and passive microwave remote sensing can detect the vertical structure of precipitation and the structural information inside the cloud, respectively. However, at present, the microwave remote sensing device is carried by the polar orbit meteorological satellite, and the time resolution is low [2]. In contrast, geostationary meteorological satellites are characterized by high spatial and temporal resolution, wide coverage, and strong time continuity, and they can effectively monitor precipitation. The cloud top brightness temperature, which is located at the highest level of the atmospheric vertical layer and is provided by the stationary meteorological satellite, can reveal significant characteristics about the existence of clouds and the evolution stage of clouds and can reflect the distribution of rain areas of typhoons to a certain extent [1].

Researchers have conducted a series of studies on satellite data retrieval of precipitation. Ferraro and Marks [4] and Alishouse et al. [5] achieved good inversion results by using statistical methods. Based on the statistical relationship between convective cloud top brightness temperature and precipitation intensity in an infrared cloud image, Lan et al. [6] developed a short-time convective precipitation intensity forecast. Moreover, Behrangi et al. [7] adopted the GOES-R satellite multichannel, PERSIANN-MSA method to estimate precipitation. They found that scheme 12 effectively recognized the presence and absence of rain with a probability of detection (POD) of 0.530 and a false-alarm ratio (FAR) of 0.355 . Similarly, based on the brightness temperature of a satellite infrared and water vapor channel, Tao et al. [8] proposed the deep neural network to estimate the precipitation and the detect the presence or absence of rain in the study area. The POD was 0.418 , and the FAR was 0.528 .

At present, precipitation retrieval algorithms focus on information from a visible, infrared window area and water vapor channel. For example, Himawari-8 remote sensing data contain other infrared channel information that can improve the accuracy of precipitation retrieval. Moreover, Min et al. [9] used random forests (RF) to carry out precipitation retrieval based on a numerical model weather forecast index and GPM and H8 data and obtained good results. Sun et al. [2] used a lookup table to carry out a study on the $\mathrm{H} 8$ infrared brightness temperature retrieval of precipitation. The more dimensions the lookup table had, the higher the retrieval accuracy was. Ebtehaj et al. [10] proposed the shrunken locally linear embedding (SLLE) algorithm for the retrieval of precipitation, which was applied to the precipitation retrieval of TRMM data with high retrieval accuracy. Because this method only uses satellite brightness temperature, the operation is relatively simple. Therefore, Wang et al. [11] applied this algorithm to the precipitation retrieval of $\mathrm{H} 8$ infrared data.

In summary, this paper focuses on Typhoon Maria and applies the retrieval algorithm proposed by Ebtehaj et al. to the infrared brightness temperature data of Advanced Geosynchronous Radiation Imager (AGRI) data from Feng-Yun 4A satellite $[12,13]$ in order to explore the role of AGRI data in typhoon precipitation monitoring. Furthermore, the information from a typhoon's dominant cloud system was extracted from the AGRI infrared window channel brightness temperature image based on the fuzzy $c$-means (FCM) clustering method $[13,14]$ to monitor the generation and change of typhoons and potentially eliminate them [15].

\section{Materials and Methods}

2.1. Precipitation Retrieval Algorithm Based on Improved Shrunken Locally Linear Embedding. This section briefly describes the satellite retrieval precipitation algorithm; the algorithm details can be found in the study of Ebtehaj et al. $[10,16]$. Satellite channel brightness temperature retrieval precipitation can be defined as a mathematical inverse problem. Suppose that at a certain pixel point (field of view), brightness temperature is observed in $n_{c}$ channels denoted by $\mathbf{y}=\left(y_{1}, y_{2}, \ldots, y_{n_{c}}\right)^{T} \in \mathfrak{R}^{n_{c}}$, and the corresponding $n_{r}$ level precipitation profile is denoted by $\mathbf{x}=$ $\left(x_{1}, x_{2}, \ldots, x_{n_{r}}\right)^{T} \in \mathfrak{R}^{n_{r}}$. The superscript $T$ indicates the vector transpose. The satellite observed brightness temperature and precipitation profiles have the following nonlinear mapping relationship:

$$
\mathbf{y}=H(\mathbf{x})+\mathbf{v},
$$

where $H: \mathbf{x} \longrightarrow \mathbf{y}$ represents the forward projection function and $\mathbf{v} \in \mathfrak{R}^{n_{c}}$ represents the model or measurement error.

The historical sample set used in this paper was defined as a matching dictionary of "brightness temperature" and "precipitation," denoted by $D=\left\{\left(\mathbf{b}_{i}, \mathbf{r}_{i}\right)\right\}_{i=1}^{M}$. $i$ is the $i$ th "atoms" in the dictionary; it could also be called the $i$ th sample. Each set of matches in the dictionary was the basic "atom" of the channel observation brightness temperature retrieval of the precipitation field. Moreover, $\mathbf{b}_{i}=\left(b_{1 i}\right.$, $\left.b_{2 i}, \ldots, b_{n_{c} i}\right) \in \mathfrak{R}^{n_{c}}$ and $\mathbf{r}_{i}=\left(r_{1 i}, r_{2 i}, \ldots, r_{n_{c} i}\right) \in \mathfrak{R}^{n_{r}}$ represent the brightness temperature of $n_{c}$ channels and the $n_{r}$-dimensional precipitation profile, respectively. The dictionary entries on "brightness temperature" and "precipitation" were denoted as $\mathbf{B}=\left[\mathbf{b}_{1}|\ldots| \mathbf{b}_{M}\right] \in \mathfrak{R}^{n_{c} \times M}$ and $\mathbf{R}=\left[\mathbf{r}_{1}|\ldots| \mathbf{r}_{M}\right] \in \mathfrak{R}^{n_{r} \times M}$, respectively.

The basic idea of the precipitation retrieval model proposed by Ebtehaj et al. [10] is to look for a group of "atoms" in the dictionary $\mathbf{B}=\left[\mathbf{b}_{1}|\ldots| \mathbf{b}_{M}\right] \in \mathfrak{R}^{n_{c} \times M}$ to represent the brightness temperature of the pixel (field of view, FOV) to be inverted and then perform the following calculation: 


$$
\mathbf{y}=\mathbf{B}_{S} \mathbf{c}+\mathbf{v},
$$

where $\mathbf{B}_{S}$ is a subset of the dictionary composed of a found set of "atoms"; $\mathbf{c}$ is the atomic coefficient; and $\mathbf{v}$ is the observation error.

By applying the atomic coefficient $\mathbf{c}$ to the matched subset of precipitation dictionary $\mathbf{R}_{s}$, we obtained the precipitation retrieval $\mathbf{x}$ as follows:

$$
\mathbf{x}=\mathbf{R}_{S} \mathbf{c}+\mathbf{e},
$$

where $\mathbf{R}_{S}$ is the subset of $\mathbf{R}=\left[\mathbf{r}_{1}|\ldots| \mathbf{r}_{M}\right] \in \mathfrak{R}^{n_{r} \times M}$ dictionary corresponding to $\mathbf{B}_{S}$; $\mathbf{c}$ is the atomic coefficient of the combined precipitation subdictionary; and $\mathbf{e}$ is the error.

Equations (2) and (3) convert the problem into a solution for atomic coefficient $\mathbf{c}$.

Based on the algorithm of Ebtehaj et al. [10], the atomic coefficient $\mathbf{c}$ can be obtained by minimizing the objective function constrained by the regular term of L1 norm, as follows:

$$
\left\{\begin{array}{l}
\underset{\mathbf{c}}{\operatorname{minimize}}\left\|\mathbf{W}^{(1 / 2)}\left(\mathrm{y}-\mathbf{B}_{S} \mathbf{c}\right)\right\|_{2}^{2}+\lambda_{1}\|\mathbf{c}\|_{1}+\lambda_{2}\|\mathbf{c}\|_{2}^{2}, \\
\mathbf{c} \geq 0,1^{T} \mathbf{c}=1,
\end{array}\right.
$$

where $\mathbf{W}$ is the contribution rate of channel observation of brightness temperature to objective function. Moreover, $\lambda_{1}$ and $\lambda_{2}$ are regularization parameters; L1 and L2-norm are $\|\mathbf{c}\|_{1}=\sum_{i}\left|c_{i}\right|$ and $\|\mathbf{x}\|_{\mathbf{A}}^{2}=\mathbf{x}^{\mathrm{T}} \mathbf{A} \mathbf{x}$, respectively, and $\mathbf{A}$ represents a positive definite matrix.

Since the L1 norm is not differentiable at the origin, equation (4) is a nonsmooth convex problem. Moreover, the L1 norm proved to be an effective method to solve sparse solutions, i.e., a small number of atoms retained the maximum amount of original information [17].

The key to the algorithm is precipitation signal identification. Compared with nonprecipitation, precipitation falls in the probability category of a "small event." Therefore, before precipitation retrieval, the $K$-nearest neighbor (KNN) method was adopted to identify the channel brightness temperature "precipitation" and "nonprecipitation" signals. The specific process was as follows: for a given channel brightness temperature $\mathbf{y}=\left(y_{1}, y_{2}, \ldots, y_{n_{c}}\right)$ to be inverted into a dictionary pair $(\mathbf{B}, \mathbf{R})$, we searched for two "optimal" joint subdictionaries $\left(\mathbf{B}_{s} \in R^{n_{c} \times K}, \mathbf{R}_{s} \in R^{n_{r} \times K}\right)$ based on KNN. Given the probability threshold predefined value $p$, based on the "probability vote" method detection, if there was more than $p_{k}$ "precipitation" in the precipitation dictionary, the channel brightness temperature contained the "precipitation" signal.

In the "precipitation signal" subspace, the representation coefficient $\widehat{\mathbf{c}}$ was obtained by solving equation (4), and the precipitation profile $\widehat{\mathbf{x}}=\mathbf{R}_{S} \widehat{\mathbf{c}}$ was obtained by satellite brightness temperature inversion. In the equation, $\widehat{\mathbf{x}}$ is the approximation of $\mathbf{x}$.

The improvement to the method proposed by Ebtehaj et al. $[10,16]$ is as follows:

(1) The brightness temperature gradient information of "precipitation" and "nonprecipitation" from the infrared spectrum of AGRI, which was obtained by statistics, was taken as the prior information and coupled to the $K$-nearest neighbor (KNN) model.
(2) The contribution rate $\mathbf{W}$ of the brightness temperature at different spectral channels to the objective function of the retrieval model (see formula (4)) was "optimized" by the Bayesian model averaging (BMA) that could be implemented with the Markov Chain Monte Carlo (MCMC) method [18, 19].

(3) Relevant parameters in the KNN model (such as the detection probability $p$ and the neighborhood $K$ ) were optimized, and the two regularization parameters of $\lambda_{1}$ and $\lambda_{2}$ were also optimized [10].

(4) The membership degree information of the dominant cloud system in AGRI typhoon, which was obtained by the fuzzy $c$-means (FCM) clustering algorithm, was applied to the precipitation retrieval. Thus, the main structure of typhoon precipitation could be acquired [13].

2.2. Fuzzy c-Means Clustering Model. The fuzzy c-means (FCM) algorithm was used to divide the data sample set into several categories by minimizing a quadratic objective function [14]. Each pixel in the image was classified individually into the corresponding category, and the minimum sum of the error squares of each sample and the cluster center were adopted as the discriminant criterion. The clustering center and membership value were searched iteratively to obtain the minimum value of the objective function so as to realize the optimal classification of the image. The FCM objective function was defined as follows:

$$
J_{m}=\sum_{i=1}^{c} \sum_{j=1}^{n} u_{i j}^{m} d^{2}\left(x_{j}, v_{i}\right)
$$

The constraint conditions of equation (5) are as follows:

$$
\sum_{i=1}^{c} u_{i j}=1, \quad 1 \leq j \leq n
$$

where $p$ is the dimension of each "feature" vector $x_{j} ; n$ is the number of feature vectors; $u_{i j}$ is the membership degree of the $j$ data belonging to the $i$ category; $m$ is the fuzzy index ( $m=2$ in this study); $v_{i}$ is the $i$ fuzzy clustering center; and $c$ is the number of categories.

The Euclidean norm was used to measure the distance between the "feature" vector $x_{j}$ and the clustering center $v_{i}$, as follows:

$$
d^{2}\left(x_{j}, v_{i}\right)=\left\|x_{j}-v_{i}\right\|^{2}
$$

According to the Lagrange extremum method, through derivation, the fuzzy membership $u_{i j}$ and clustering center $v_{i}$ were obtained as follows:

$$
\left\{\begin{array}{l}
u_{i j}=\left(\sum_{k=1}^{c}\left(\frac{d\left(x_{j}, v_{i}\right)}{d\left(x_{j}, v_{k}\right)}\right)^{(2 /(m-1))}\right)^{-1}, \\
v_{i}=\frac{\sum_{j=1}^{n} u_{i j}^{m} x_{j}}{\sum_{j=1}^{n} u_{i j}^{m}} .
\end{array}\right.
$$




\section{Precipitation Signal Identification Based on FY4A/AGRI Infrared Channel Brightness Temperature}

3.1. Data Description. China's new geostationary FY4A satellite was successfully launched on December 11, 2016. The FY4A/AGRI has 14 channels, including three visible, three near-infrared, and eight infrared $[12,13]$. Because only the AGRI infrared channels ranging from 8 to 14 were assimilated in the numerical weather prediction system $[12,13]$, we only considered the brightness temperatures of these seven channels for typhoon precipitation retrieval. Their central wavelengths were $3.72,6.25,7.10,8.5,10.8,12$, and 13.5 micron (unit: $\mu \mathrm{m}$ ), respectively. The time interval between two consecutive images of the AGRI brightness temperature was $15 \mathrm{~min}$ during observation encryption, and only integral data at hourly times such as 0000 UTC, 0100 UTC, and so on were used in this paper.

The domain of the Global Precipitation Measurement (GPM) IMERG precipitation data used in this investigation covered $89.95^{\circ} \mathrm{S}-89.95^{\circ} \mathrm{N}$ latitude and $179.95^{\circ} \mathrm{W}-179.95^{\circ} \mathrm{E}$ longitude, with a spatial resolution of $0.1^{\circ} \times 0.1^{\circ}[13,20]$. The time resolution was hourly $(\mathrm{mm} / \mathrm{h})$, i.e., 24 precipitation observation times per day. The GPM IMERG data are available at https://pmm.nasa.gov/data-access/downloads/gpm.

Due to the different spatial resolutions of FY4A/AGRI and GPM data, when constructing the dictionary of the historical sample base, the "nearest neighbor" method was adopted to interpolate the channel brightness temperature of FY4A/ AGRI to the GPM FOV. For evaluation of the precipitation retrieval accuracy, the "nearest neighbor" method was adopted to interpolate the retrieval precipitation to the GPM FOV.

3.2. Background of Typhoon Maria. Supertyphoon Maria (international code: 1808) was the eighth storm of the 2018 typhoon season in the Pacific Ocean. At 05:00 Coordinated Universal Time (UTC), July 8, 2018, Maria had developed into a supertyphoon in the northwest Pacific Ocean and was located approximately $1,930 \mathrm{~km}$ to the southeast of Yilan County, Taiwan. At 09:10 UTC, July 11, 2018, Maria made landfall on the Huang Qi peninsula of Lian Jiang, Fujian province. The maximum wind force near the center reached level $14(42 \mathrm{~m} / \mathrm{s})$, and the lowest pressure was $960 \mathrm{hPa}$. At 11: 00 UTC, July 11, eight cities in the Fujian province and 48 counties (cities and districts) in Pingtan were affected; 187,600 people were affected, and 154,500 were displaced [13]. The study area was $15.35^{\circ} \mathrm{N}-38.75^{\circ} \mathrm{N}, 112.35^{\circ} \mathrm{E}-139.75^{\circ} \mathrm{E}$.

3.3. Precipitation Signal Identification Based on the AGRI Infrared Brightness Temperature. The brightness temperature of AGRI infrared channel in the region of Typhoon Maria on July 09,2018 , to July 11,2018 , was used to identify precipitation signals. The matching GPM data were used to judge the precipitation FOVs. The FY4A/AGRI brightness temperature gradient $(\triangle \mathrm{TB}$, unit: $\mathrm{K})$, defined as the difference between the brightness temperature of nonprecipitation and precipitation (unit: K) in different channels, is shown in Figure 1(a).
In order to analyze the influence of different precipitation intensification on brightness temperature of AGRI channel, five grades of precipitation were further counted. The precipitation grades are nonprecipitation $[0,0.1),[0.1,5)$, [5-10), [10-15), and $[15,+\infty) \mathrm{mm} / \mathrm{h}$ (denoted as "15-Inf"), respectively.

For AGRI channels with different grades of precipitation, the mean brightness temperature (TB Mean, unit: K) is shown in Figure 1(b).

Figure 1(a) reveals that for FY4A/AGRI infrared channels 8 to 14 , when precipitation occurred, the brightness temperature gradient of the channels all changed. Channel 9 underwent the smallest change range, at only $13.93 \mathrm{~K}$, and channel 12 had the largest range change, at $40.28 \mathrm{~K}$.

Figure 1(b) reveals that even with only weak precipitation, AGRI channel's brightness temperature variation range was still large. When the precipitation intensity increased, the change range of the brightness temperature gradient in the AGRI channel was small, which may be related to the small number of precipitation samples in the statistical history sample database and the insufficient representation. It may be also related to the infrared channel's sensitivity to clouds, which can generally only detect cloud top brightness temperature and cannot penetrate clouds [21]. Precipitation is related to cloud top height, cloud top temperature, and cloud vertical structure. Channels of different wavelengths (the peak layer of the weight function varies; see Figure 2(b)) can reflect different aspects of clouds. Therefore, the responses of the brightness temperatures of different AGRI channels to the precipitation signal vary (Figure 1(a)). Precipitation can be successfully retrieved using the combined information of brightness temperatures from different channels. Generally, the cloud bodies of precipitation clouds are thicker, the cloud tops are higher, and the cloud top temperatures are lower. Thus, cold cloud tops generally correspond to heavy precipitation intensity, and the responses of different channels to different levels of precipitation vary (Figure 1(b)).

\section{Results and Discussion}

4.1. Optimization of Channel Contribution Rate Based on $B M A$. In this paper, the "precipitation signal" of the AGRI infrared channel was obtained based on the $K$-nearest neighbor (KNN) approach. Based on BMA, we estimated the contribution rate of different channels of AGRI to the objective function of the retrieval model. In the optimization process, BMA-Gamma, BMA-Normal, and BMA-Heteroscedastic were adopted to obtain the channel contribution rates, which are shown in Figure 2(a). Gamma, Normal, and Heteroscedastic represent the conditional probability distributions of Heteroscedastic Gamma, Homoscedastic Normal, and Heteroscedastic Normal, respectively [18].

We adopted radiative transfer for the Tiros Operational Vertical Sounder [22] to determine the weight function of the AGRI channels from 8 to 14 by calculating the standard American atmospheric profile, as shown in Figure 2(b).

Figure 2(a) reveals that the contribution rate of channel 9 (the weight function peak was $358.28 \mathrm{hPa}$, as shown in 


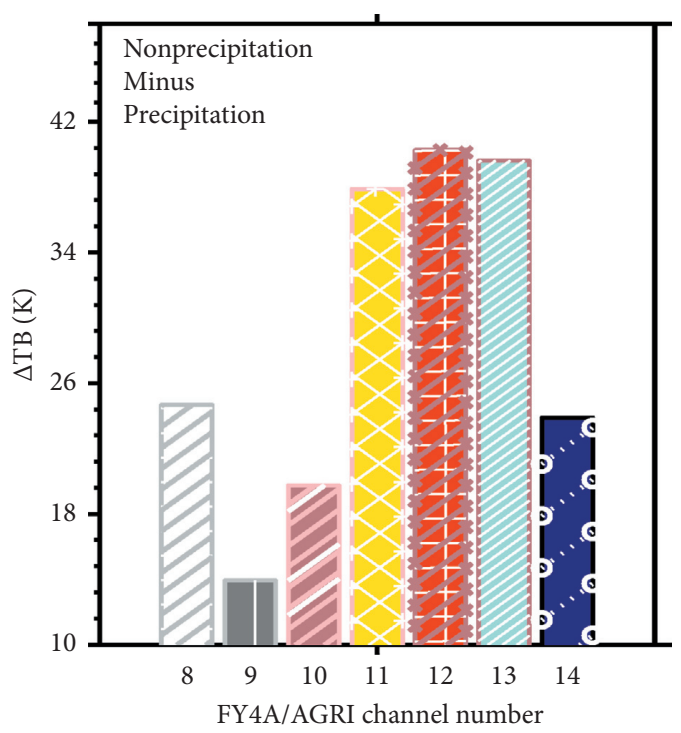

(a)

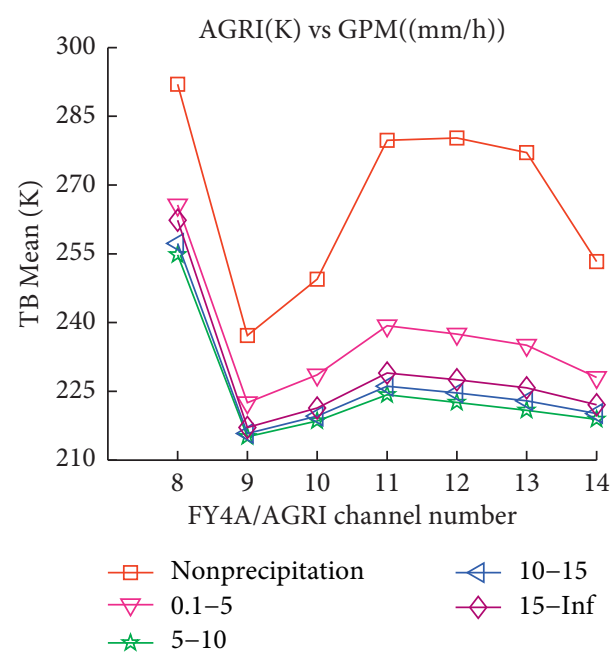

(b)

FIGURE 1: Brightness temperature gradient of nonprecipitation and precipitation (a) and brightness temperature average of different precipitation grades (b).
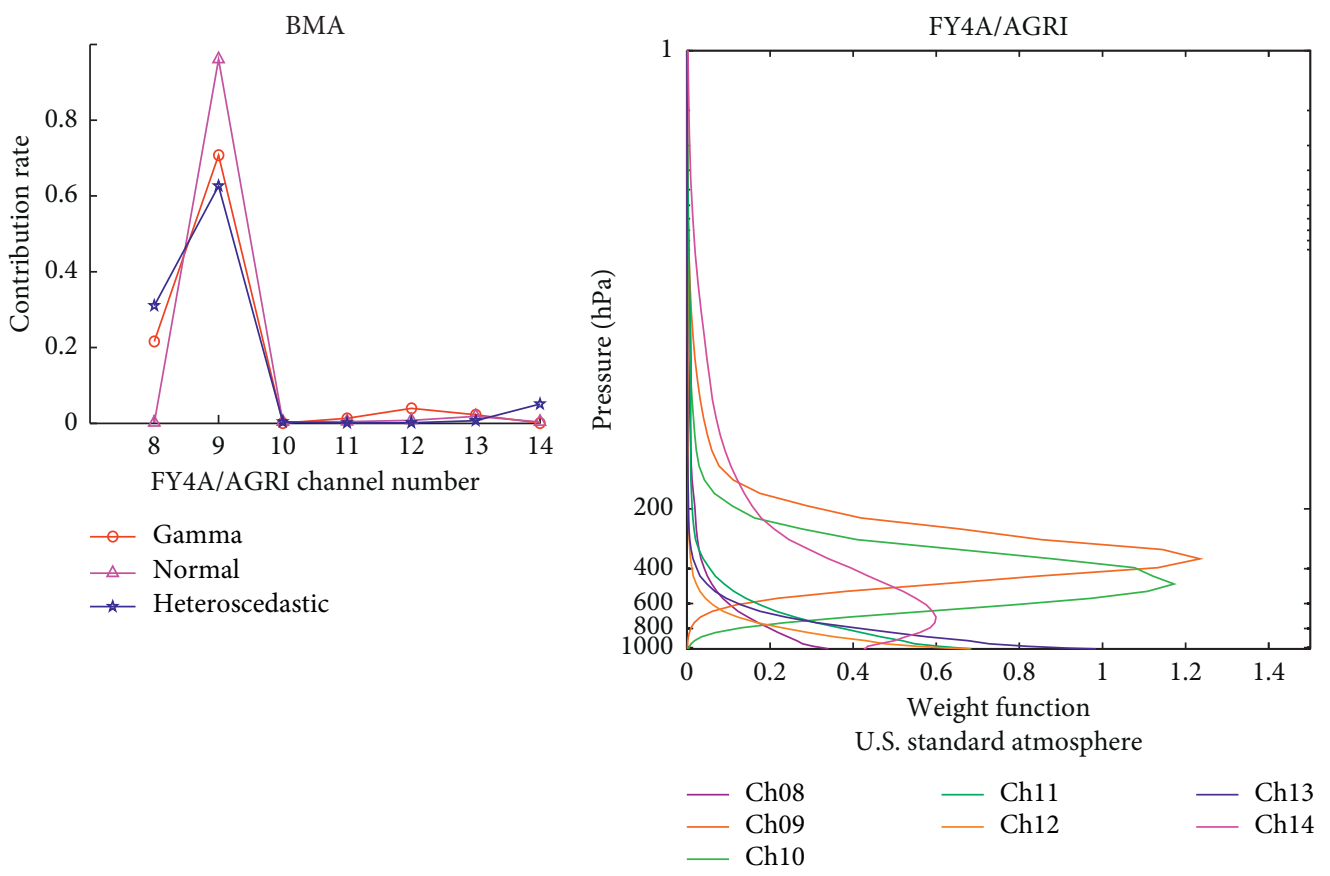

(a)

(b)

FIGURE 2: Contribution rate of AGRI channel brightness temperature optimization based on the BMA method (a) and the weight function distribution of AGRI channels 8 to 14 (b).

Figure 2(b)) based on BMA-Gamma was large, which may be related to channel 9's main detection of atmospheric water vapor. The contribution value of other channels was small.

The contribution rates of different AGRI channels obtained based on BMA-Gamma were better than those in the precipitation retrieval, which may be related to the characteristics of the "Gamma" distribution of precipitation [23].

\subsection{Application Study on Precipitation Retrieval in Typhoon Maria}

4.2.1. Dominant Cloud System Identification and Tracking Based on the FCM Method. The key to solving the problem of cloud system extraction is to identify the "dominant cloud system," which often leads to typhoon precipitation [15]. In order to analyze the structure of a typhoon's dominant cloud 
system, we used the FCM to identify the dominant cloud system based on the 10.8-micron window channel of the AGRI. Specific details on the identification of the dominant cloud system can be found in the work of Wang et al. [13].

Figure 3 shows the original brightness temperature of AGRI and the dominant cloud system based on the FCM and GPM precipitation distribution in the formation of Typhoon Maria.

As shown in Figure 3, the brightness temperature distribution of typhoon's dominant cloud system identified by the FCM was consistent with the GPM precipitation field, and the AGRI brightness temperature low value area corresponded to the strong precipitation area of GPM. This may be related to the inherent characteristics of the FCM method. The typhoon spiral cloud band structure in the original AGRI brightness temperature image has not been effectively identified $[13,15]$, which may be related to the inherent characteristics of the FCM method. A great deal of information concerning a typhoon's motion, wind field, and heavy rainfall is contained in its spiral cloud bands. Moreover, based on the GPM precipitation field (time: 2018071016), we know that GPM data can reflect the "spiral rain belt" of a typhoon.

4.2.2. Accuracy Assessment Measurement of AGRI Retrieval Precipitation of Typhoon Maria. We adopted the following quantitative evaluation indices to assess the accuracy of precipitation retrieval: structural similarity (SSIM), normalized mutual information (NMI) [24], peak signal-tonoise ratio (PSNR) [17], probability of detection (POD), false-alarm ratio (FAR), and critical success index (CSI) $[13,25]$.

Comparing the precipitation field $U$ and $V$, SSIM is defined as

$$
\operatorname{SSIM}(u, v)=\frac{\left(2 \overline{u v}+c_{1}\right)\left(2 \overline{(u-\bar{u})(v-\bar{v})}+c_{2}\right)}{\left(\overline{u^{2}}+\overline{v^{2}}+c_{1}\right)\left(\overline{(u-\bar{u})^{2}}+\overline{(v-\bar{v})^{2}}+c_{2}\right)},
$$

where $c_{1}$ and $c_{2}$ are two constants to stabilize the computation; $u$ and $v$ are partial datasets of precipitation field $U$ and $V$, respectively; and $\bar{u}$ and $\bar{v}$ are the mean values of selected neighborhoods (typically $5 \times 5$ and $7 \times 7$ FOVs, etc.) around a certain FOV of $u$ and $v$, respectively. The overbar $(-)$ indicates the mean value.

Defining the peak signal-to-noise ratio (PSNR):

$$
\operatorname{PSNR}=20 \log \left(\frac{L}{\sqrt{\mathrm{MSE}}}\right),
$$

where $L$ refers to the dynamic range of the GPM precipitation field. MSE $=\overline{(U-V)^{2}}$, in which $U$ and $V$ are GPM and retrieval precipitation field, respectively.

$$
\begin{aligned}
\mathrm{POD} & =\left(\frac{A}{(A+B)}\right), \\
\mathrm{FAR} & =\left(\frac{C}{(A+C)}\right), \\
\mathrm{CSI} & =\left(\frac{A}{(A+B+C)}\right),
\end{aligned}
$$

where $A$ is the number of FOVs in which both $U$ and $V$ are smaller than the threshold; $B$ is the number of FOVs in which $U$ is smaller than the threshold while $V$ is greater than the threshold; and $C$ is the number of FOVs in which $U$ is greater than the threshold while $V$ is smaller than the threshold. The threshold is set as $5.0 \mathrm{~mm} / \mathrm{h}$ in this paper.

4.2.3. Precipitation Retrieval Experimental of AGRI Infrared Channel Brightness Temperature Based on an Improved Local Linear Embedding Method. In this study, the brightness temperature obtained from AGRI channels 8-14 was used to retrieve precipitation. The precipitation field at different development stages of Typhoon Maria retrieved with the improved SLLE method is shown in Figure 4. Moreover, to get the main structure of typhoon precipitation, taking the membership degree of the dominant cloud system from the AGRI channels as the prior information, which was obtained by the FCM clustering algorithm, the modified precipitation retrieval result (denoted as "precipitation retrieval (FCM)") could be acquired.

The observed data (namely, "true value") in Figure 4 corresponds to the GPM precipitation at different times in Figure 3.

Moreover, the accuracy assessment on the precipitation of "Typhoon Maria" retrieved from AGRI is shown in Table 1.

As shown in Figure 4 and Table 1, the retrieval precipitation field based on the improved SLLE method agreed with GPM and successfully retrieved the spiral cloud rain bands of the typhoon. In addition, the precipitation field after the membership degree modification of the dominant cloud system based on FCM could identify the main morphological structure of typhoon precipitation, especially in high-precipitation value areas, but the precipitation information of the spiral cloud rain belt of "typhoon" was excessively removed [15]. It can be seen that (formulas (9)-(13)), compared with the retrieval precipitation field obtained by the improved SLLE method, part of the field of view (FOV) values was eliminated (that is, the FOV value was assigned to 0 ) in the precipitation field obtained by the FCM modification. Therefore, the retrieval result by the FCM modification exhibits a decrease in the SSIM, NMI, and PSNR values and an increase in the POD and FAR values, whereas there is a decrease in the CSI value, as shown in 


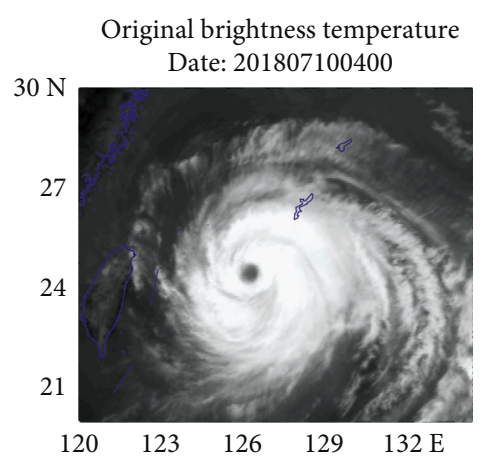

(a)

Original brightness temperature

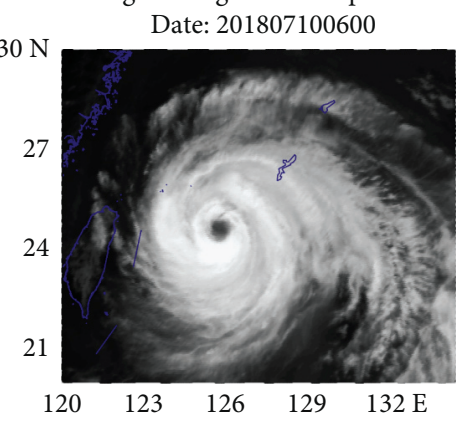

(d)

Original brightness temperature

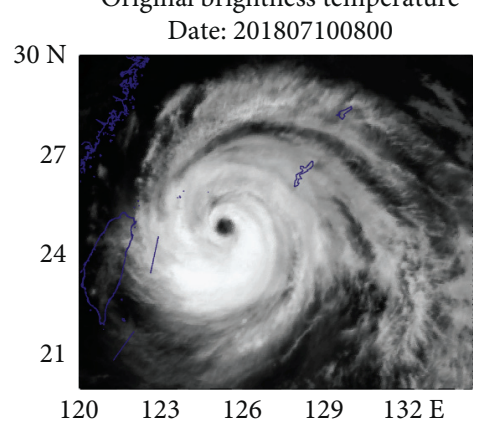

(g)

Original brightness temperature

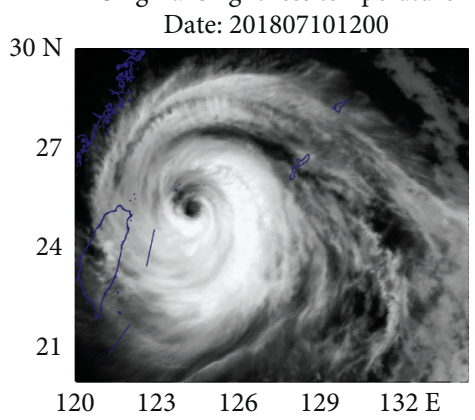

(j)

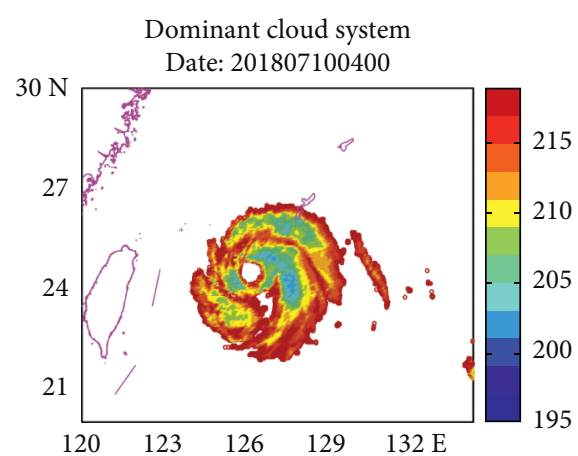

(b)

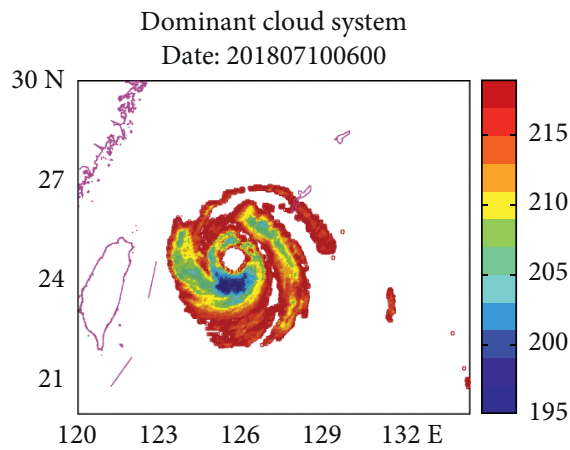

(e)

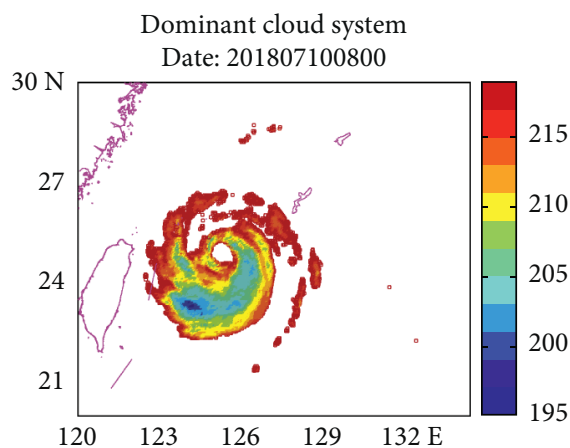

(h)

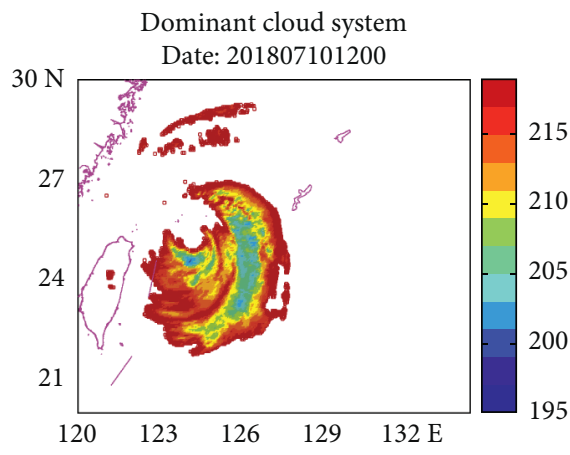

(k)
GPM

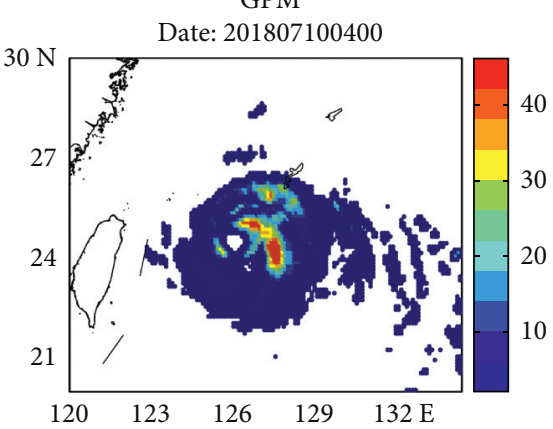

(c)

GPM

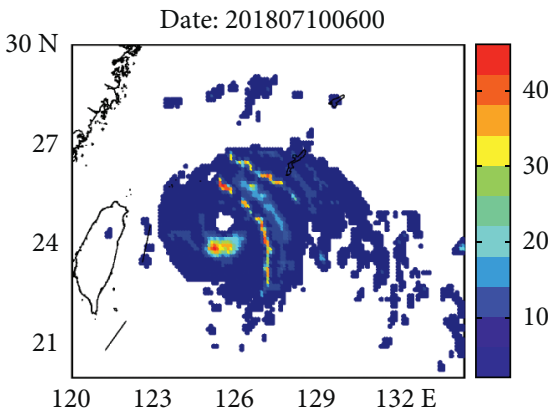

(f)

GPM

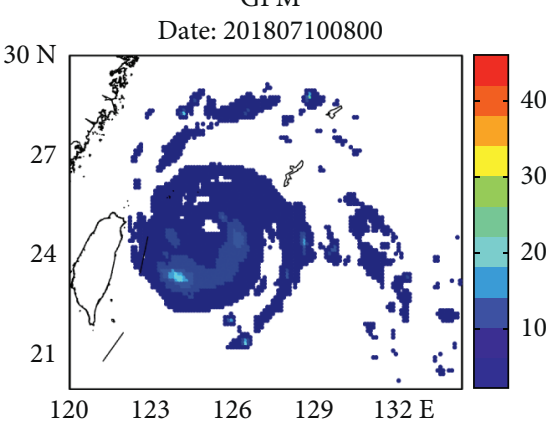

(i)

GPM

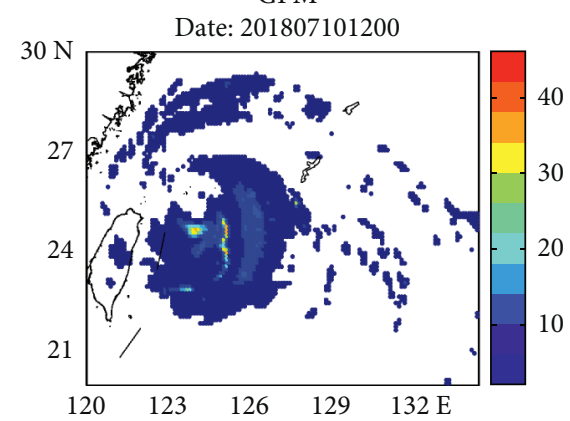

(1)

Figure 3: Continued. 


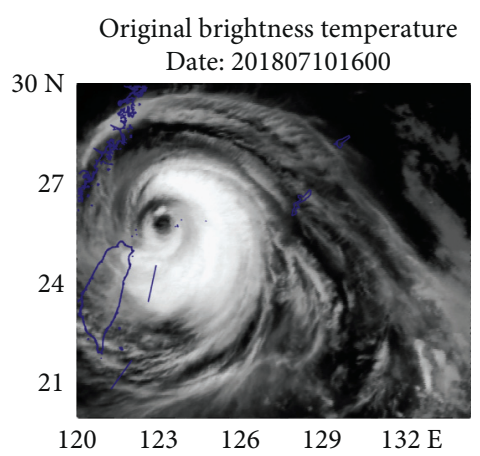

(m)

Original brightness temperature Date: 201807101800

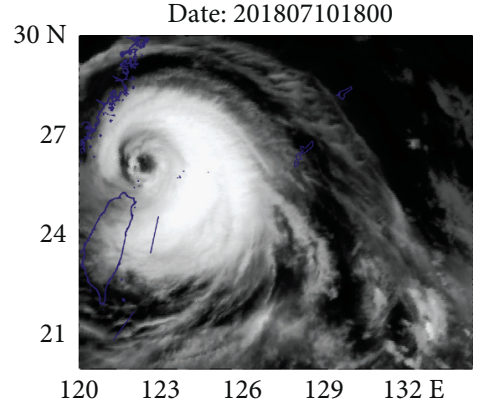

(p)

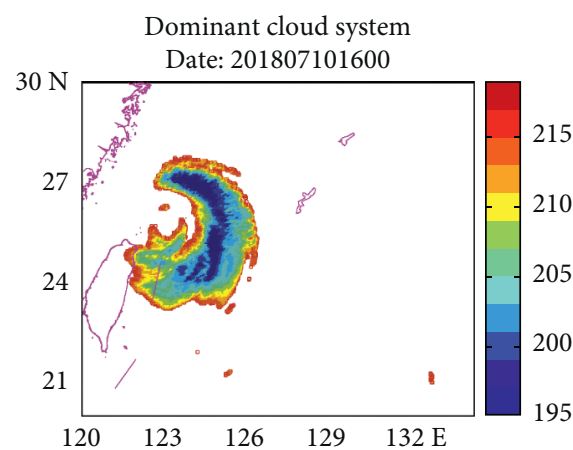

(n)

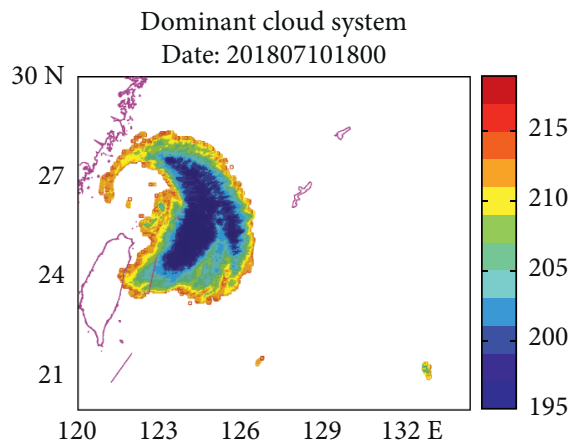

(q)
GPM

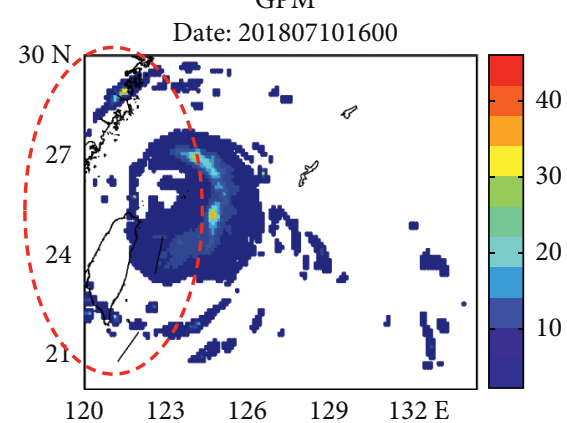

(o)

GPM

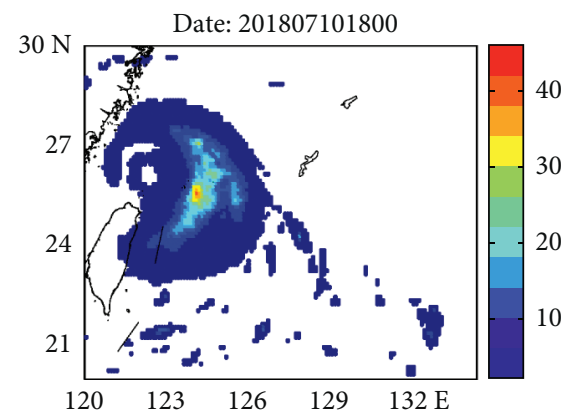

(r)

FIGURE 3: Sequence changes of AGRI original brightness temperature (a, d, g, j, m, p), dominant cloud system based on FCM (b, e, h, k, n, q), and GPM precipitation (c, f, I, l, o, r).

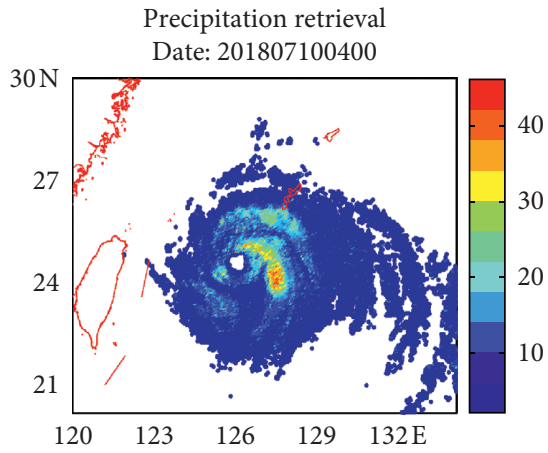

(a)

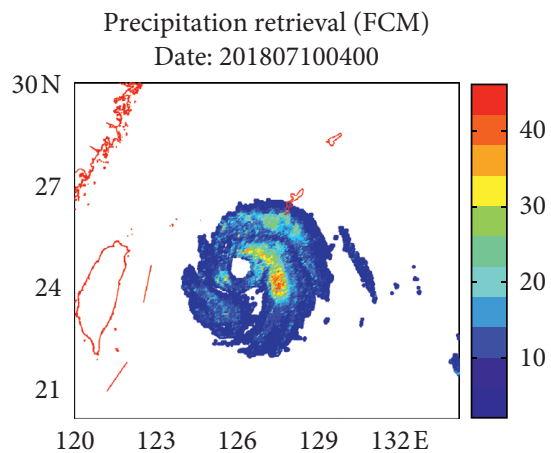

(d)

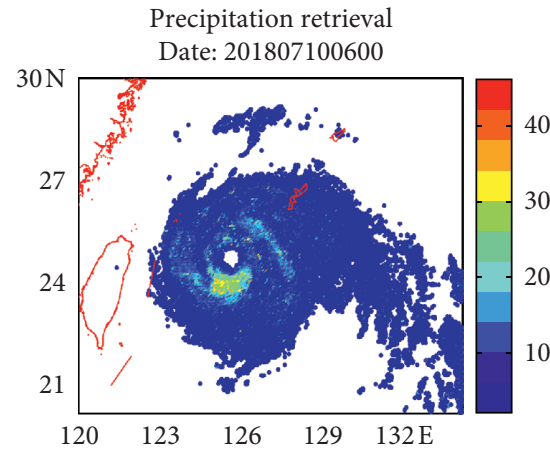

(b)

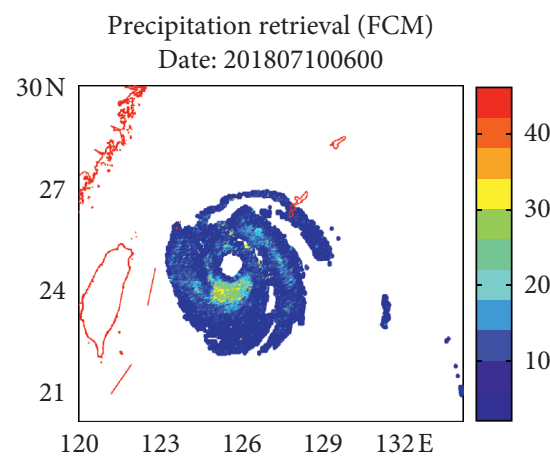

(e)

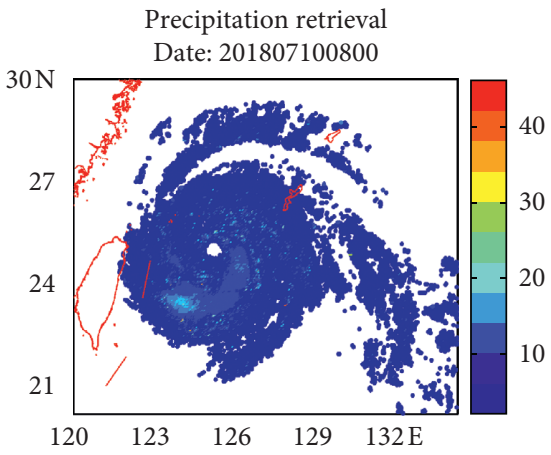

(c)

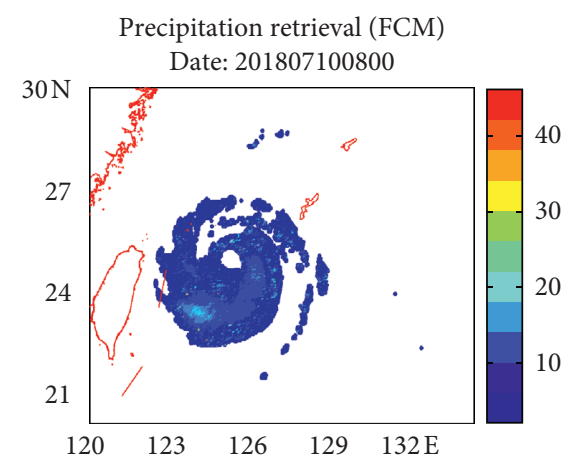

(f)

Figure 4: Continued. 


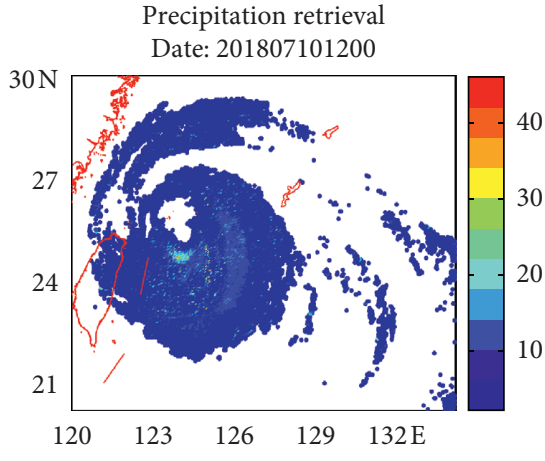

(g)

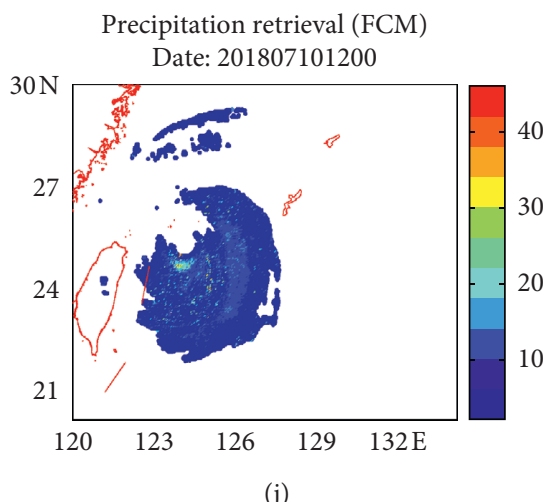

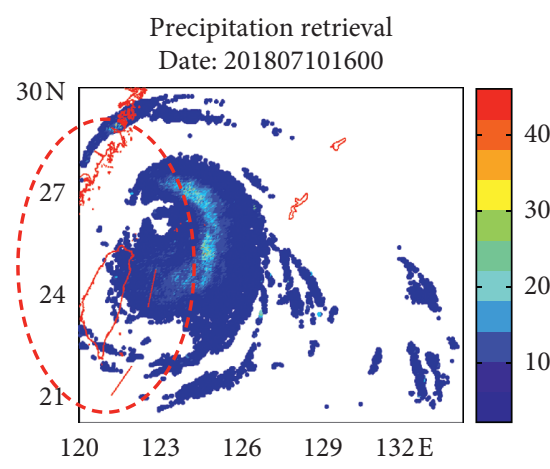

(h)

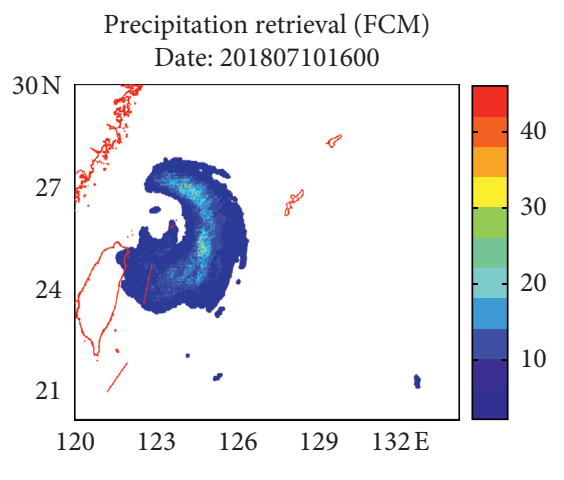

(k)

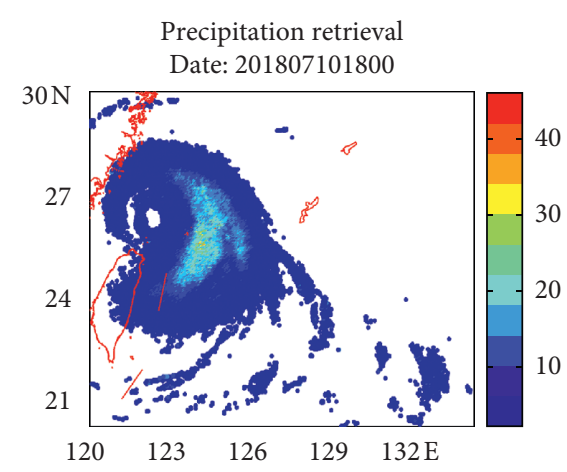

(i)

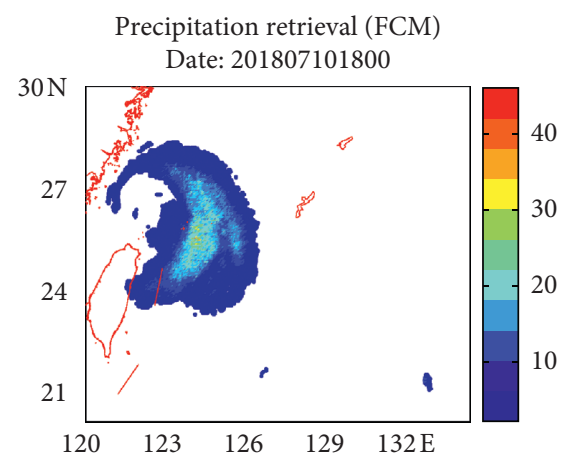

(l)

FIGURE 4: Precipitation retrieval of AGRI based on the SLLE method (a, b, c, g, h, i) and the dominant cloud system membership degree of FCM (d, e, f, j, k, l).

TABLE 1: Comparison of AGRI retrieval precipitation and GPM during Typhoon Maria.

\begin{tabular}{lcccccc}
\hline & $\begin{array}{c}\text { SSIM SLLE/ } \\
\text { SLLE + FCM }\end{array}$ & $\begin{array}{c}\text { NMI SLLE/ } \\
\text { SLLE + FCM }\end{array}$ & $\begin{array}{c}\text { PSNR SLLE/ } \\
\text { SLLE + FCM }\end{array}$ & $\begin{array}{c}\text { POD (\%) SLLE/ } \\
\text { SLLE + FCM }\end{array}$ & $\begin{array}{c}\text { FAR (\%) } \\
\text { SLLE/SLLE + FCM }\end{array}$ & $\begin{array}{c}\text { CSI (\%) } \\
\text { SLL/SLLE + FCM }\end{array}$ \\
\hline 2018071004 & $0.902 / 0.881$ & $0.355 / 0.171$ & $28.385 / 26.734$ & $99.38 / 99.62$ & $1.17 / 1.61$ & $98.22 / 98.02$ \\
2018071006 & $0.888 / 0.857$ & $0.338 / 0.133$ & $27.606 / 24.847$ & $99.31 / 99.54$ & $1.23 / 1.7$ & $98.09 / 97.86$ \\
2018071008 & $0.892 / 0.863$ & $0.299 / 0.121$ & $27.875 / 22.649$ & $99.54 / 99.69$ & $0.81 / 1.11$ & $98.74 / 98.58$ \\
2018071012 & $0.957 / 0.937$ & $0.272 / 0.165$ & $31.813 / 30.428$ & $99.86 / 99.90$ & $0.35 / 0.46$ & $99.51 / 99.44$ \\
2018071016 & $0.957 / 0.941$ & $0.222 / 0.139$ & $31.693 / 30.476$ & $99.95 / 99.97$ & $0.38 / 0.47$ & $99.57 / 99.49$ \\
2018071018 & $0.965 / 0.946$ & $0.276 / 0.170$ & $33.142 / 32.590$ & $99.98 / 99.98$ & $0.39 / 0.43$ & $99.60 / 99.55$ \\
\hline
\end{tabular}

Note. The SSIM unit is dimensionless. The larger the SSIM, the more similar the 2 comparison objects. When the SSIM is 1 , the 2 comparison objects are the same. The unit of NMI is mm, and the unit of PSNR is dB. The larger the PSNR value is, the less distortion there is between the retrieval field and the actual GPM field. Precipitation thresholds in POD, FAR, and CSI were all set to $5.0 \mathrm{~mm} / \mathrm{h}$.

Table 1 . This may be related to the inherent characteristics of the FCM method, which we will study in greater detail in the future.

\subsubsection{Analysis of Time Series Changes of Dominant Cloud} System Development of Typhoon Maria Based on the FCM Method. In this study, FCM was used to identify the dominant cloud system of typhoons with a brightness temperature in the AGRI 10.8-micron window channel, and the time series changes of the typhoon's dominant cloud system were measured through radiation parameters (cloud top average brightness temperature and minimum brightness temperature) and shape parameters (cloud area) [26].
The cloud area was defined as the number of pixel points (FOVs) in this paper.

Figure 5 shows the FCM dominant cloud system identification results from 0000 UTC to 1800 UTC on July 10, 2018, and the time series changes of cloud top average brightness temperature (denoted as TB Mean; unit: K), minimum brightness temperature (denoted as TB Min; unit: $\mathrm{K})$, cloud area (denoted as Area (FOVs)), and average brightness temperature gradient (denoted as $\triangle \mathrm{TB}$ Mean; unit: K).

As shown in Figure 5, at 1600 UTC on July 10, 2018, the typhoon's dominant cloud system had the largest change in the mean brightness temperature, the minimum brightness temperature was $194.1 k$, and the cloud system contained the 


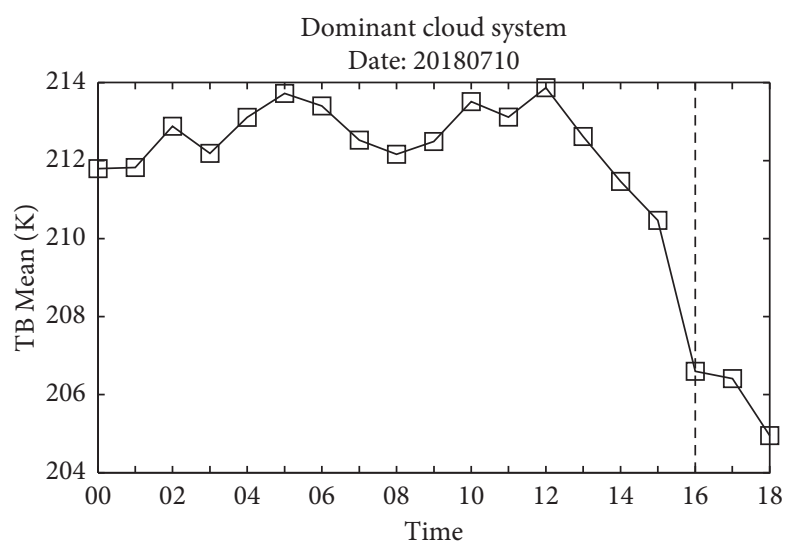

(a)

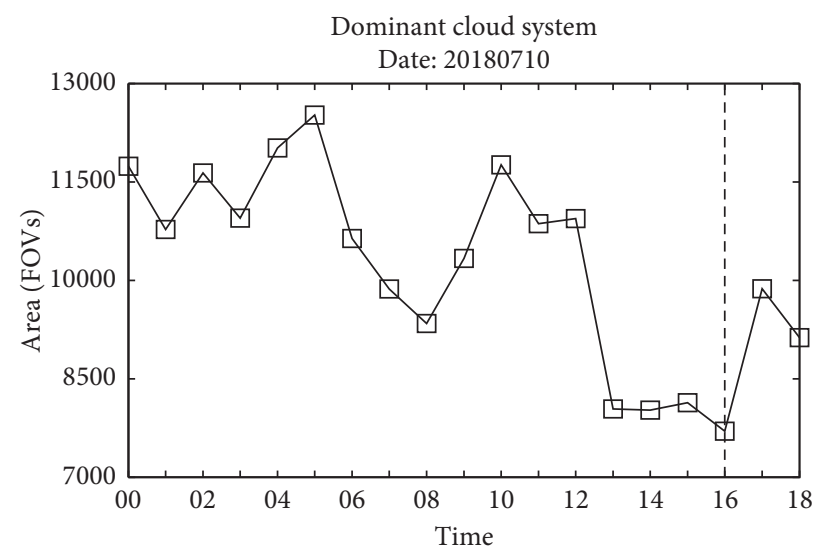

(c)

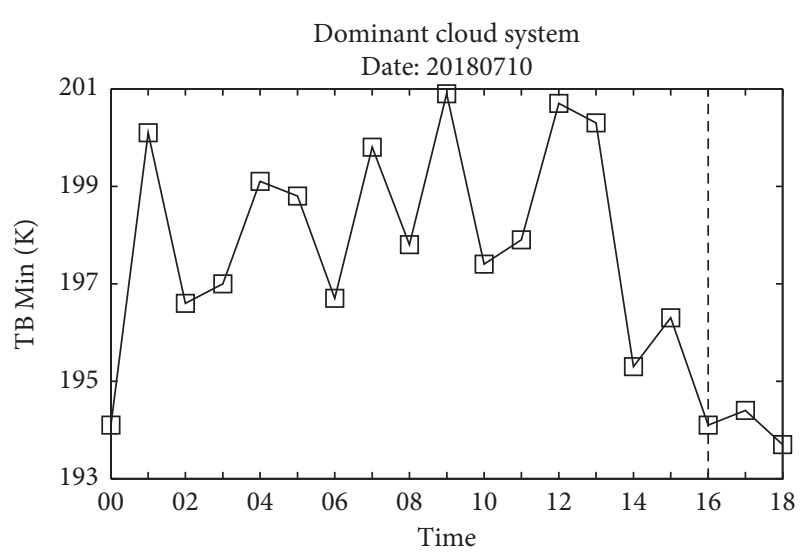

(b)

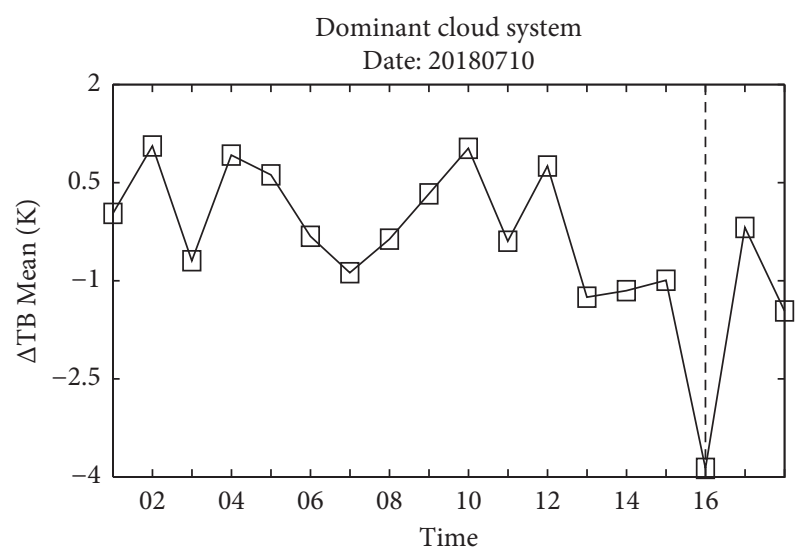

(d)

FIgURE 5: Temporal variation of radiation parameters ( $a, b)$, shape parameters (c), and gradient (d) of AGRI cloud cluster.

smallest pixel (FOVs) area. The brightness temperature gradient had the largest variation range, and there was a "jump" point. Combined with a typhoon path (website: http:// typhoon.nmc.cn/web.html) analysis, we observed the moment when the typhoon turned from a supertyphoon into a typhoon. Thus, it can be concluded that the brightness temperature gradient of the dominant cloud system identified by FCM could be used as an indicative factor to a certain extent, which is favorable for the analysis on the intensity change of typhoons. It also shows the feasibility of FY4A/ AGRI data in typhoon monitoring and precipitation retrieval.

\section{Summary and Future Work}

Retrieval of precipitation by satellite infrared data has always been a research hotspot. In this study, infrared data from Typhoon Maria in 2018 were selected and applied to typhoon precipitation monitoring based on the statistical analysis of the brightness temperature gradient in different infrared channels of "precipitation" and "nonprecipitation" FOVs by using Feng-Yun 4A/AGRI infrared channel brightness temperature.

Our main results were summarized as follows:

(1) Identification of "precipitation" and "nonprecipitation" signals based on FY4A/AGRI infrared brightness temperature: when precipitation occurred, the brightness temperature gradient of channels 8 to 14 all changed, and channel 12 had the largest change range. According to statistics on different precipitation grades, even when there is weak precipitation, the brightness temperature of each channel changes greatly, so the infrared data of AGRI have a good response to precipitation signal identification.

(2) Retrieval of typhoon precipitation information based on the improved SLEE method: the L1 norm regularization in the retrieval model can promote sparsity; that is, the maximum amount of original information can be retained with fewer dictionary atoms. From the precision evaluation index, we concluded that the typhoon precipitation retrieval field with the channel contribution rate based on BMA-Gamma is close to GPM and can retrieve the spiral rain band of a typhoon.

(3) Modification of the precipitation retrieval field based on FCM membership degree: the typhoon's dominant cloud system identified by FCM aligned with the precipitation distribution of the GPM, and the brightness temperature low-value area of AGRI corresponded to the strong precipitation area of the 
GPM. The dominant cloud system membership degree information obtained by precipitation FCM modified the precipitation retrieval field and obtained the main precipitation morphological structure of the typhoon.

(4) Dominant cloud system change analysis during the development of Typhoon Maria: based on FCM, the changes in the average brightness temperature, minimum brightness temperature area, and brightness temperature gradient of the dominant cloud system can be used to preliminarily analyze the development and change of typhoons.

In this study, only the application of the algorithm was considered. However, the period of statistical analysis data was short, there were few case studies conducted, and the historical sample base was not representative enough. In a future stage of this study, a large number of practical case studies will be carried out, starting from data mining to carry out the study on the optimal data volume of dictionary. When constructing features, numerical "model space" information, such as $K$ index and convection-effective potential energy index, will be added [25]. In addition, the texture information of the visible channel will be integrated into the infrared channel. That said, a robust statistical method was introduced into the retrieval of objective function, the Huber norm of the M-estimator was added [21], and the overall morphological structure of precipitation field was considered. The results of this study can be further applied to typhoon precipitation monitoring, forecasting, and early warning to improve meteorological services for the public.

\section{Data Availability}

The data used to support the findings of this study are available from their corresponding websites. The FY4A/ AGRI data are available at http://satellite.nsmc.org.cn/ portalsite/default.aspx?currentculture=en-US. The GPM IMERG data are available at https://pmm.nasa.gov/dataaccess/downloads/gpm.

\section{Conflicts of Interest}

The authors declare that they have no conflicts of interest.

\section{Authors' Contributions}

Gen Wang performed the experiments, analyzed the data, optimized and built the model, and drafted the manuscript. Kefu Wang provided technical support for using the precipitation retrieval method and contributed to the analysis and discussion. Wei Han and Dongyong Wang provided technical support for using the FCM method and contributed to the analysis, discussion, and editing of the manuscript. Xuexing Qiu provided important suggestions regarding the precipitation assessment of infrared data as well as manuscript editing.

\section{Acknowledgments}

This work was supported by the National Natural Science Foundation of China (grant nos. 41805080 and 41675108), the Natural Science Foundation of Anhui Province (grant no. 1708085QD89), and the Key Research and Development Program Projects of Anhui Province (grant no. 201904a07020099). The authors would like to express our sincere thanks to Dr. Ebtehaj for his precipitation retrieval based on passive microwave data codes (code available at https://www.hydsens.com/downloads). The authors would like to thank NASA and the National Satellite Meteorological Centre for freely providing the GPM IMERG and FY4A/AGRI data online. They would also like to thank LetPub (http://www.letpub.com) for its linguistic assistance during the preparation of this manuscript.

\section{References}

[1] Y.-F. Yan, J.-G. Tan, L.-L. Cui et al., "Estimating the shorttime severe precipitation of Typhoon Meranti and its evolution by using the infrared brightness temperature data form Himawari-8 satellite with high spatio-temporal resolution," Meteorological Monthly, vol. 45, no. 3, pp. 318-329, 2019, in Chinese.

[2] S.-H. Sun, W.-B. Li, and Y.-P. Huang, "Retrieval of precipitation by using Himawari-8 infrared images," Acta Scientiarum Naturalium Universitatis Pekinensis, vol. 55, no. 2, pp. 215-226, 2019, in Chinese.

[3] J.-F. Lu, R.-S. Chen, C.-T. Han et al., "Evaluating TRMM multi-satellite precipitation analysis using gauge precipitation and MODIS snow-cover products," Advances in Water Science, vol. 21, no. 3, pp. 343-348, 2010, in Chinese.

[4] R. R. Ferraro and G. F. Marks, "The development of SSM/I rain rate retrieval algorithms using ground based radar measurements," Journal of Atmospheric and Oceanic Technology, vol. 12, no. 8, pp. 755-770, 1995.

[5] J. C. Alishouse, S. A. Snyder, J. Vongsathorn, and R. R. Ferraro, "Determination of oceanic total precipitable water from the SSM/I," IEEE Transactions on Geoscience and Remote Sensing, vol. 28, no. 5, pp. 811-816, 1990.

[6] H.-P. Lan, R.-L. Zhang, and Y. Jiang, "Infrared rainfall estimation technique for small area and its use in very short-term weather forecasting," Journal of Tropical Meteorology, vol. 16, no. 4, pp. 366-373, 2000, in Chinese.

[7] A. Behrangi, K.-1. Hsu, B. Imam, S. Sorooshian, G. J. Huffman, and R. J. Kuligowski, "PERSIANN-MSA: a precipitation estimation method from satellite-based multispectral analysis," Journal of Hydrometeorology, vol. 10, no. 6, pp. 1414-1429, 2009.

[8] Y. Tao, K. Hsu, A. Ihler, X. Gao, and S. Sorooshian, "A twostage deep neural network framework for precipitation estimation from bispectral satellite information," Journal of Hydrometeorology, vol. 19, no. 2, pp. 393-408, 2018.

[9] M. Min, C. Bai, J.-P. Guo et al., "Estimating summertime precipitation from Himawari-8 and global forecast system based on machine learning," IEEE Transactions on Geoscience and Remote Sensing, vol. 57, no. 5, pp. 2557-2570, 2018.

[10] A. M. Ebtehaj, R. L. Bras, and E. Foufoula-Georgiou, "Shrunken locally linear embedding for passive microwave retrieval of precipitation," IEEE Transactions on Geoscience and Remote Sensing, vol. 53, no. 7, pp. 3720-3736, 2015. 
[11] G. Wang, C.-H. Ding, and H.-L. Liu, "Precipitation retrieval from Himawari-8 satellite infrared data based on dictionary learning method and regular term constraint," Meteorological and Environmental Research, vol. 10, no. 3, pp. 61-65, 2019.

[12] J. Yang, Z. Zhang, C. Wei, F. Lu, and Q. Guo, "Introducing the new generation of Chinese geostationary weather satellites, Fengyun-4," Bulletin of the American Meteorological Society, vol. 98, no. 8, pp. 1637-1658, 2017.

[13] G. Wang, D.-Y. Wang, W. Han, and J. Yin, "Typhoon cloud system identification and forecasting using the Feng-Yun 4A/ Advanced Geosynchronous Radiation Imager based on an improved fuzzy clustering and optical flow method," Advances in Meteorology, vol. 2019, Article ID 5890794, 11 pages, 2019.

[14] G. Wang, J.-W. Zhang, Y.-J. Chen et al., "An anisotropic GVF model for the MR image segmentation of left ventricle," Journal of Computer-Aided Design \& Computer Graphics, vol. 22, no. 11, pp. 1887-1891, 2010, in Chinese.

[15] Q. Bai and K. Wei, "Cloud system extraction in tropical cyclones by mountain-climbing," Atmospheric Research, vol. 101, no. 3, pp. 611-620, 2011.

[16] A. M. Ebtehaj and C. D. Kummerow, "Microwave retrievals of terrestrial precipitation over snow covered surfaces: a lesson from the gpm satellite," Geophysical Research Letters, vol. 44, no. 12, pp. 6154-6162, 2017.

[17] A. M. Ebtehaj, G. E. Foufoula, and G. Lerman, "Sparse regularization for precipitation downscaling," Journal of Geophysical Research: Atmospheres, vol. 117, Article ID D08107, 12 pages, 2012.

[18] A. E. Raftery, T. Gneiting, F. Balabdaoui, and M. Polakowski, "Using Bayesian model averaging to calibrate forecast ensembles," Monthly Weather Review, vol. 133, no. 5, pp. 1155-1174, 2005.

[19] J. A. Vrugt, C.-J.-F. Braak, G.-G.-H Diks et al., “Accelerating Markov chain Monte Carlo simulation by differential evolution with self-adaptive randomized subspace sampling," International Journal of Nonlinear Sciences and Numerical Simulation, vol. 10, no. 3, pp. 271-288, 2009.

[20] K.-B. Sounak and V. Chandrasekar, "Chandrasekar crossvalidation of observations between the GPM dual-frequency precipitation radar and ground based dual-polarization radars," Remote Sensing, vol. 10, no. 11, p. 1773, 2018.

[21] G. Wang, F. Tang, X.-B. Liu et al., "Application of M-estimators method on FY-3B/IRAS channel brightness temperature generalized variational assimilation," Journal of Remote Sensing, vol. 21, no. 1, pp. 52-61, 2017, in Chinese.

[22] J. Hocking, P. Rayer, D. Rundle et al., RTTOV v11 Users Guide. NWPSAF-MO-UD-028, Met Office, Exeter, UK, 2015, https://nwpsaf.eu/site/download/documentation/rtm/docs_ rttov11/users_guide_11_v1.4.pdf.

[23] G. J. Husak, J. Michaelsen, and C. Funk, "Use of the gamma distribution to represent monthly rainfall in Africa for drought monitoring applications," International Journal of Climatology, vol. 27, no. 7, pp. 935-944, 2007.

[24] O. K. Tarald, "On normalized mutual information: measure derivations and properties," Entropy, vol. 19, no. 11, p. 631, 2017.

[25] Z. Liu, M. Min, J. Li et al., "Local severe storm tracking and warning in pre-convection stage from the new generation geostationary weather satellite measurements," Remote Sensing, vol. 11, no. 4, p. 383, 2019.

[26] Y.-A. Liu, M. Wei, W. Gao, and N. Li, "Short-term automatic forecast algorithm of severe convective cloud identification using FY-2 IR images," Journal of Remote Sensing, vol. 16, no. 1 , pp. 72-92, 2012, in Chinese. 